\title{
Propagação de ondas ultrassônicas elásticas em modelos sintéticos com heterogeneidades esféricas
}

\author{
Rafael S. G. Gomes* (Universidade Federal do Pará, Brasil), José J. S. de Figueiredo (Universidade Federal do Pará, Brasil \& \\ INCT-GP, Brasil), Lucas R. A. Nunes(Faculdade de Geofísica-UFPA) e Thiago P. Prestes (Faculdade de Geofísica-UFPA)
}

Copyright 2018, SBGf - Sociedade Brasileira de Geofísica

This paper was prepared for presentation during the $8^{\text {th }}$ Brazilian Symposium on Geophysics, held in Salinópolis, Brazil, 18 to 20 september 2018.

Contents of this paper were reviewed by the Technical Committee of the $8^{\text {th }}$ Brazilian Symposium on Geophysics and do not necessarily represent any position of the SBGf, its officers or members. Electronic reproduction or storage of any part of this paper for commercial purposes without the written consent of The Brazilian Geophysical Society is prohibited.

\section{Abstract}

The manufacture of synthetic rocks samples has a great importance in the study of the elastic properties of the rocks based on the variation of heterogeneities. In this present work we constructed synthetic sandstones with different number of heterogeneities in the samples. In this work we constructed and analyzed (in dry condition and satured condition) thr samples were constructed in this work. In total, twelve heterogeneous samples and an isotropic sample for reference. The heterogeneous samples were divided in three groups (A, B and C). Group A with heterogeneities of $3.75-4 \mathrm{~mm}$ diameter group $\mathbf{B}$ with heterogeneities of $5-6 \mathrm{~mm}$ diameter and group C with heterogeneities of $6.5-7.5 \mathrm{~mm}$. From P- and S-waveforms were measured $\mathrm{Vp}$ and Vs velocities. From the $P$ and $S$ waveforms $V p$ and $V$ velocities were measured with these results, we compare the experimental velocities with the theoretical models, which is the Maxwell-Garnett and Kuster-Toksoz model, thus predicting the velocities $P$ and $S$ for the dry and saturated cases. As expected the results were satisfactory with low percentage error and proving that these two models are optimal to predict the speeds before this type of sample.

\section{Introdução}

O estudo da física de rochas é essencial quando se trata da prospecção de hidrocarbonetos, principalmente quando os reservatórios são aqueles formados por rochas porosas o suficiente para conter fluidos (água, gás e óleo). Como é conhecido estas rochas são chamadas de rochas reservatórios. O arenito é um dos grandes exemplos de rocha reservatório, tanto de petróleo quanto de gás, e essa acumulação ocorre entre os grãos das rochas, ou na heterogeneidades, fissuras ou fraturas (Barwis et al., 1990).

Utilizando modelos sintéticos heterogêneos (construídos de forma controlada), pode-se correlacionar as propriedades físicas com as velocidades de ondas compressionais e cisalhantes. A variação de porosidade secundária (relacionado a espalhadores, fraturas, fissuras, etc) influência na velocidade, na qual as ondas ultrassônicas atravessam as rochas o que permite saber qual é a distribuição de heterogeneidades no reservatório, visto que quanto maior a porosidade, menor a velocidade das ondas e consequentemente, maior a chance de ter recursos naturais dentro das rochas reservatórios neste local de alta porosidade S. and Korringa (1975). Portanto, o estudo da física das rochas é muito importante para o entendimento de suas propriedades e os fluidos presentes nos poros, isso permite ter interpretações para relacionar particularidades sísmicas com os parâmetros conhecidos no reservatório para prospectar hidrocarbonetos.

Neste trabalho, a metodologia aplicada na produção das amostras sintéticas foi criada por Santos et al. (2017). Nele é simulado um arenito heterogêneo sintético com uma certa concentração de areia com cimento. Esse método é bastante importante para a modelagem física, pois tem um material similar comparando as propriedades físicas de um arenito real (Santos et al., 2016). No total 13 amostras foram construídas e analisadas em condição, doze delas com heterogeneidades e uma isotrópica homogênea. As amostras heterogêneas foram divididas em três grupos $(A, B$ e $C$ ) de quatro amostras (cada grupo possui um tamanho diferente de heterogeneidade). Resultados mostram que para ambos os grupos as velocidades das ondas $P$ e $S$ decrescem com o aumento do número de espalhadores. No entanto, os dados das velocidades experimentais é comparado com velocidades de modelos teóricos (Maxwell-Garnett e Kuster-Toksoz), e com isso, apresenta uma excelente previsão dos dados experimentais.

\section{Metodologia}

A construção dos modelos heterogêneos e as medidas ultrassônicas foram realizadas no Laboratory of Petrophysics and Rock Physics-Dr. Om Prakash Verma (LPRP), na Universidade Federal do Pará (UFPA).

\section{Construção das amostras}

O processo de produção dos modelos sintéticos começa a partir da determinação de três grupos contendo quatro amostras em cada um. Elas são subdivididas nos grupos no tipo A,B e $C$ e uma amostra de referência sem heterogeneidades. Esses grupos serão subdivididos pela quantidade de heterogeneidades, e também, pelo diâmetro médio das heterogeneidades de cada grupo de amostras, no qual foram 4, 5,5 e 7,00 $\mathrm{mm}$, respectivamente. A partir da determinação destes fatores, o passo a seguir foi determinar a porcentagem de areia peneirada e cimento para satisfazer a amostra sintética de estudo, no qual foi 
$30 \%$ de cimento e $70 \%$ de areia para a simulação de um arenito (com porosidade média primária de 19\%), essa mistura com uma certa quantidade de água forneceu uma consistência na mistura para ser colocada ao vasilhame. Realizando estes processos, foi utilizado um vasilhame (cano) de $37.56 \mathrm{~mm}$ de diâmetro e cerca de $60 \mathrm{~mm}$ de altura para alocar a mistura e as heterogeneidades, o processo de alocação das heterogeneidades teve o auxílio de um outro cano com 20,48 $\mathrm{mm}$. Esse serviu para que as mesmas ficassem bem centralizadas e que não ocorresse 0 risco de ir para as laterais, as Figuras 1 e 2 mostra esse processo. Com isso, foram feitos os modelos mostrados na Figura 3, subdivididos em grupos, o primeiro foi o grupo $A$ contendo as amostras $A-10, A-20, A-30$ e $A-40$, grupo $B$ subdividas em $B-10, B-20, B-30$ e $B-40$, grupo $C$ subdividido em $C-10, C-20, C-30$ e $C-40$ e por fim, uma amostra de referência sem heterogeneidades. Os números correspondentes aos lados das letras são os números de heterogeneidades colocadas em cada amostra. Após o processo de alocação da massa, as amostras ficaram durante 48 horas para a secagem em temperatura ambiente, esse tempo fez com que a amostra tivesse uma solidificação sem a criação de fissuras. Após a solidificação estas amostras foram colocada em um líquido com o objetivo de diluir todas as heterogeneidades e restando apenas espaço vazios esféricos. Essa técnica foi desenvolvida por Santos et al. (2017) para criação de modelos heterogêneos e anisotrópicos. As figuras abaixo mostram as etapas do processo de confecção dos modelos.

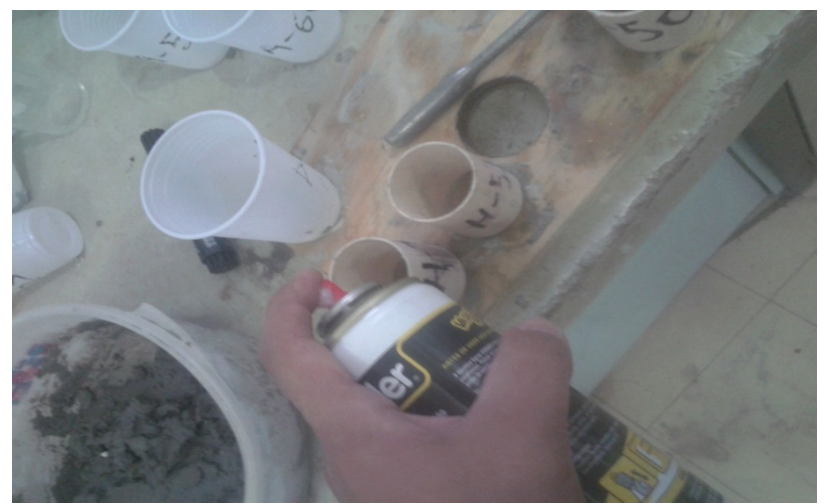

Figura 1: This picture, shows the vaseline spray being passed in the bottle to make it easy to remove the sample.

O próximo passo a seguir, foi a realização de medidas, tais como:comprimento, massa seca, massa saturada, densidade, porosidade e volume (ver a tabelas 1 e 2 para os valores medidos). Foi utilizado uma balança analítica e um paquímetro, e assim, realizando as medidas de massa (seca e saturada) e comprimento. Obtendo essas informações, foi possível estimar da porosidade de cada amostra confeccionada (de uma maneira aproximada). A porosidade (ver as tabelas 1, 2 e 3 para os valores medidos) e foi estimada por:

$$
\begin{gathered}
M_{\text {agua }}=M_{\text {saturada }}-M_{\text {Seca }} \\
\rho_{\text {agua }}=1=\frac{M_{\text {agua }}}{V_{\text {agua }}}
\end{gathered}
$$

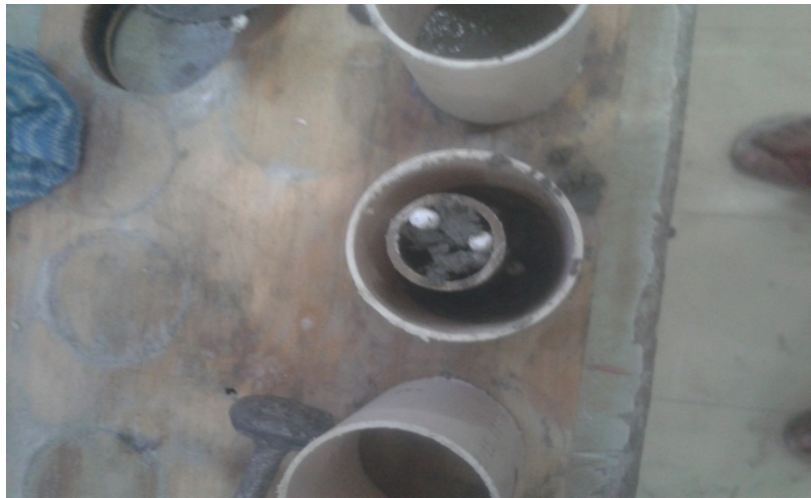

Figura 2: The use of the other pipe guaraante the location of the heterogeneities inside of the sample.

$$
\begin{array}{r}
V_{\text {total }}=\pi r^{2} h \quad(\text { Da amostra }) \\
\text { Porosidade }(\phi)=\frac{V_{\text {agua }}}{V_{\text {total }}},
\end{array}
$$

no qual $r$ e $h$ são o raio e altura do plug enquanto $\mathrm{M}$ é a massa da amostra (seca ou saturada). Para evitar contaminação de impurezas, na equação 1 o tipo de água utilizada foi a destilada.

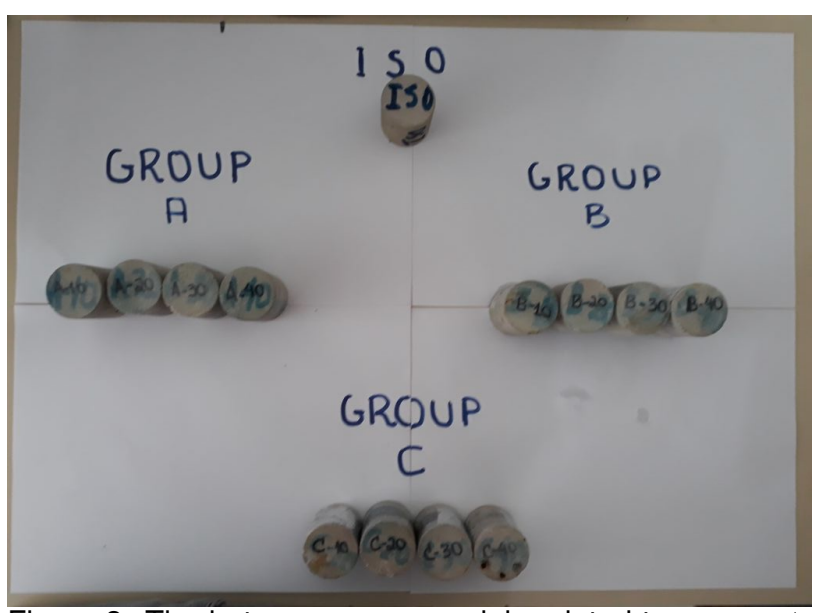

Figura 3: The heterogeneous models related to groups $A$, $B, C$ and the reference model.

Foram feitas amostras ilustradas na Figura ?? para mostrar como as heterogeneidades se comportam dentro da amostra, e também, mostrar os espaços vazios deixados no processo de dissolução pelos respectivos grupos.

\section{Modelos teóricos (Maxwell-Garnett e Kuster-Toksoz)}

O modelo de Maxwell-Garnett é uma extensão da relação de Clausius-Mossotti, no qual relaciona uma propriedade macroscópica (constante dielétrica $(\varepsilon)$ ) em uma propriedade microscópica (polarizabilidade molecular $(\alpha)$ ). Para o modelo de Maxwell-Garnett será assumido um modelo para a inclusão, pois não há exigência para uma ligação definitiva com a teoria microscópica. Sem essa exigência com a teoria microscópica, será modificada as propriedades físicas do sistema microscópico para o 
macroscópico (granular). A partir disto, será realizada a mudança empirica das propriedades elétricas para propriedades elásticas, assim, obtendo o módulo efetivo de incompressibilidade e cisalhamento.

$$
\varepsilon=\varepsilon_{0}+\frac{3 \eta_{1} \gamma_{1}}{1-\eta_{1} \gamma_{1}} \varepsilon_{0}
$$

which

$$
\gamma_{1}=\frac{\varepsilon_{1} \varepsilon_{0}}{\varepsilon_{1}+2 \varepsilon_{0}}
$$

$\varepsilon_{0} \rightarrow$ dieletric constant of material without inclusion.

$\varepsilon_{1} \rightarrow$ dieletric constant of inclusions.

$\eta_{1} \rightarrow$ volumetric fraction of the molecules -(inclusions).

$$
\begin{gathered}
\varepsilon=\varepsilon_{0}+\frac{3 \eta_{1} \gamma_{1}}{1-\eta_{1} \gamma_{1}} \varepsilon_{0} \rightarrow K_{\text {eff }}=K_{\text {iso }}+\frac{3 \eta_{i} \gamma_{i}}{1-\eta_{i} \gamma_{i}^{K}} K_{\text {iso }} \\
\varepsilon=\varepsilon_{0}+\frac{3 \eta_{1} \gamma_{1}}{1-\eta_{1} \gamma_{1}} \varepsilon_{0} \rightarrow \mu_{\text {eff }}=\mu_{\text {iso }}+\frac{3 \eta_{i} \gamma_{i}}{1-\eta_{i} \gamma_{i}^{\mu}} \mu_{\text {iso }} \\
\gamma_{1}=\frac{\varepsilon_{1} \varepsilon_{0}}{\varepsilon_{1}+2 \varepsilon_{0}} \rightarrow \gamma_{i}^{K}=\frac{K_{\text {fluid }}-K_{\text {iso }}}{K_{\text {fluid }}+2 K_{\text {iso }}} ; \gamma_{i}^{\mu}=\frac{\mu_{\text {fluid }}-\mu_{\text {iso }}}{\mu_{\text {fluid }}+2 \mu_{\text {iso }}} ; \\
\eta_{i}=N_{i} \frac{V_{\text {incl }}}{V_{\text {tot }}} ;
\end{gathered}
$$

$K_{i s o}=$ Bulk modulus of the reference sample;

$K_{\text {eff }}=$ Effective Bulk modolus;

$\mu_{\text {eff }}=$ Effective Shear modulus;

$\eta=$ fracture density;

O modelo de Kuster-Toksoz utiliza a teoria da dispersão do comprimento de onda de primeira ordem para buscar expressões de velocidade $\mathrm{P}$ e $\mathrm{S}$ a partir dos módulos efetivos de incompressibilidade e cisalhamento para qualquer forma de inclusão. Outras constantes são importantes para incorporar nas equações dos módulos efetivos, essas constantes são especificas para cada tipo de onda, tanto compressional como cisalhante dependendo apenas do tipo de inclusão .

$$
\begin{aligned}
\mu_{K T}^{e f f} & =\frac{\mu_{i s o}\left(\mu_{i s o}+\zeta_{m}\right)+\zeta_{m}\left(\eta_{i n c}\left(\mu_{i n c}-\mu_{i s o}\right) Q^{m i}\right)}{\left(\mu_{i s o}+\zeta_{m}\right)-\eta_{i n c}\left(\mu_{i n c}-\mu_{i s o}\right) Q^{m i}} \\
K_{K T}^{e f f} & =\frac{K_{i s o}\left(K_{i s o}+\frac{4}{3} \mu_{i s o}\right)+\frac{4}{3} \mu_{i s o} \eta_{i n c}\left(K_{i n c}-K_{i s o}\right) P^{m i}}{K_{\text {iso }}+\frac{4}{3} \mu_{i s o}-\eta_{\text {inc }}\left(K_{\text {inc }}-K_{\text {iso }}\right) P^{m i}}
\end{aligned}
$$

\section{Medidas ultrassônicas}

As aquisições de dados ultrassônicos foram realizadas utilizando o sistema ultrassônico do LPFR. Foi utilizada a técnica de transmissão de pulso ultrassônicos (Santos et al., 2016). O sistema do LPFR é formado por um gerador e receptor de pulso 5072PR e um pré-amplificador $5660 B$, ambos da Olympus, um osciloscópio USB de $50 \mathrm{MHz}$ da Handscoope, e quatro transdutores. Sendo dois de $1 \mathrm{MHz}$ (Onda $\mathrm{P}$ ) e dois $500 \mathrm{kHz}$ (Onda S), também da Olympus. A Figura 4 mostra esses equipamentos citados acima.

A taxa de amostragem por canal para todas as medidas das formas de onda P e S foi de $0.01 s$. Para a medição

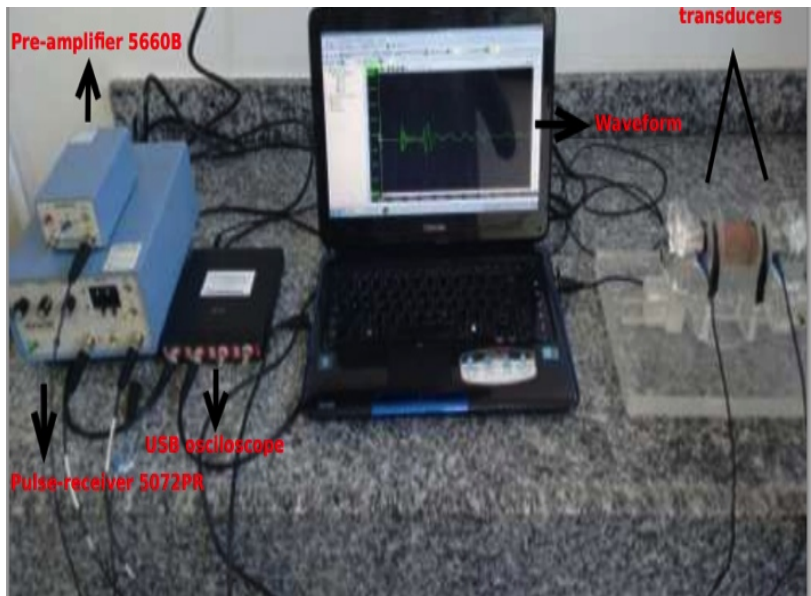

Figura 4: This figure shows two transducers (source and receiver) of $S / P$ wave and the other equipment's that compose the ultrasonic system (pulse-receiver and preamplifier).

os transdutores ficaram separados pelo comprimento do modelo. Os transdutores foram centralizados nas amostras para assegurar que a propagação de ondas estava na região desejada, pois as heterogeneidades estão bem no centro dos modelos. Isto foi feito tanto para as aquisições das ondas $\mathrm{P}$ e $\mathrm{S}$, os dados ultrasssônicos coletados nos modelos foram o tempo de chegada das ondas $\mathrm{P} / \mathrm{S}$.

\section{Resultados}

A partir da aquisição dos traços, foi realizado o tratamento dos dados adquiridos através do sistema ultrassônico no LPRP, esse tratamento foi realizado no ferramenta matemática computacional MatLab. Devido a presença de ruído inerente do sistema, foi aplicado uma suavização nos dados para retirada de ruídos aleatórios e ruídos de rede. O gráfico da Figura 11 mostra apenas o traço sísmico para o grupo da amostras A. Foi realizado uma picagem manual dos tempos de chegadas das ondas P e S. Nas tabelas 1, 2 e 3 estão mostrados os tempos de trânsito (para ondas $P$ e S) para cada amostra e grupo. Para as medidas experimentais, foi aplicado uma barra de erro para realizar a comparação com as velocidades teóricas de MaxwellGarnett e Kuster-Toksoz, no qual a porcentagem de erro aplicado foi: $(0.05,0.05,0.07,0.07$ e 0.07$)$, para os modelo do grupo incluido também a amostra de referência. A partir dos tempos de chegadas dos traços sísmicos e com os valores do comprimento e do tempo pode-se encontrar a velocidade das ondas $P$ e $S$ (também mostradas nas tabelas 1, 2 e 3).

O comprimento médio utilizado no grupo $A$ foi cerca de $55.75 \mathrm{~mm}$ na qual foram moldados para que ficasse dentro dessa média, para não influenciar na porosidade e na velocidade para que não haja discordâncias na hora de expor os gráficos, outro é que a densidade fica entre 1.83 e $1.79\left(\frac{g}{\mathrm{~cm}^{3}}\right)$ dentro do limite desejado para a simulação do arenito. As velocidades $\mathrm{P}$ calculadas pelo tempo de chegada, verifica-se o decaimento da velocidade por conta do aumento de heterogeneidades na amostra, e também, é possivel perceber o aumento das velocidades $\mathrm{P}$ quando a amostra fica saturada em relação ao caso seco, já a velocidade da onda $S$ decai também com o 
aumento da porosidade, e também, na comparação do caso seco e saturado, a velocidade $\mathrm{S}$ no caso seco é maior do que no caso saturado por conta da água não resistir ao cisalhamento. As velocidades da onda $P$ e $S$ teóricos (modelos de Maxwell-Garnett e Kuster-Toksoz) e experimentais podem ser verificadas nas Figuras 5 e 6 .

Tabela 1: The table shows the measurements performed in-group $A$ of the heterogeneous models, performed in LPRP.

\begin{tabular}{|c|c|c|c|c|}
\hline Group A & $A-10$ & $A-20$ & $A-30$ & $A-40$ \\
\hline Lenght $(\mathrm{mm})$ & 57.44 & 54.34 & 55.04 & 56.20 \\
\hline $\operatorname{Mass}_{d r y}(g)$ & 115.86 & 108.09 & 109.03 & 110.67 \\
\hline $\operatorname{Mass}_{\text {satured }}(g)$ & 128.83 & 120.44 & 121.67 & 123.80 \\
\hline Porosity $(\phi)$ & 0.205 & 0.207 & 0.209 & 0.213 \\
\hline Volume $\left(\mathrm{cm}^{3}\right)$ & 63.00 & 59.60 & 60.37 & 61.63 \\
\hline Density $_{\text {dry }}\left(\frac{g^{\prime}}{\mathrm{cm}^{3}}\right)$ & 1.839 & 1.813 & 1.805 & 1.795 \\
\hline Density $_{\text {sat }}\left(\frac{\mathrm{g}}{\mathrm{cm}^{3}}\right)$ & 2.044 & 2.020 & 2.015 & 2.008 \\
\hline time $_{p \text { dry }}(s)$ & 20.87 & 19.91 & 20.36 & 20.95 \\
\hline time $_{s}$ dry $(s)$ & 29.99 & 28.78 & 29.56 & 30.73 \\
\hline time $_{p \text { sat }}(s)$ & 18.17 & 17.42 & 17.82 & 18.42 \\
\hline time $_{s \text { sat }}(s)$ & 31.50 & 29.95 & 30.39 & 31.17 \\
\hline$V p_{d r y}(m / s)$ & 2733.93 & 2710.39 & 2685.04 & 2664.61 \\
\hline$V s_{d r y}(m / s)$ & 1906.40 & 1879.09 & 1853.81 & 1820.42 \\
\hline$V p_{\text {sat }}(\mathrm{m} / \mathrm{s})$ & 3137.08 & 3094.72 & 3064.77 & 3027.83 \\
\hline$V s_{\text {sat }}(\mathrm{m} / \mathrm{s})$ & 1815.42 & 1806.02 & 1802.92 & 1794.84 \\
\hline
\end{tabular}

Tabela 2: The measurements performed in-group B of the heterogeneous models, performed in LPRP.

\begin{tabular}{|c|c|c|c|c|}
\hline Group B & $B-10$ & $B-20$ & $B-30$ & $B-40$ \\
\hline Lenght $(\mathrm{mm})$ & 56.52 & 55.57 & 54.62 & 58.85 \\
\hline $\operatorname{Mass}_{d r y}(g)$ & 110.25 & 105.58 & 101.80 & 107.50 \\
\hline $\operatorname{Mass}_{\text {satured }}(g)$ & 123.50 & 118.95 & 115.30 & 122.34 \\
\hline Porosity $(\phi)$ & 0.213 & 0.219 & 0.225 & 0.229 \\
\hline Volume $\left(\mathrm{cm}^{3}\right)$ & 61.99 & 60.95 & 59.90 & 64.55 \\
\hline Density $_{d r y}\left(\frac{g^{\prime}}{\mathrm{cm}^{3}}\right)$ & 1.778 & 1.732 & 1.699 & 1.665 \\
\hline Density $_{\text {sat }}\left(\frac{g}{\mathrm{~cm}^{3}}\right)$ & 1.992 & 1.951 & 1.924 & 1.895 \\
\hline time $_{p}$ dry $(s)$ & 20.72 & 20.84 & 20.87 & 22.82 \\
\hline time $_{s}$ dry $(s)$ & 30.07 & 30.47 & 31.05 & 34.34 \\
\hline time $_{p \text { sat }}(s)$ & 18.17 & 18.31 & 18.45 & 20.27 \\
\hline times sat $_{(s)}$ & 31.73 & 32.12 & 32.57 & 35.81 \\
\hline$V p_{d r y}(m / s)$ & 2709.65 & 2648.71 & 2599.55 & 2563.29 \\
\hline$V s_{d r y}(\mathrm{~m} / \mathrm{s})$ & 1871.01 & 1815.41 & 1751.09 & 1706.88 \\
\hline$V p_{\text {sat }}(m / s)$ & 3087.01 & 3011.92 & 2937.95 & 2883.55 \\
\hline$V s_{\text {sat }}(\mathrm{m} / \mathrm{s})$ & 1773.32 & 1722.47 & 1669.30 & 1636.71 \\
\hline
\end{tabular}

A Mesma analogia feita para o grupo $A$, será feito para o grupo $B$ e para o grupo $C$, pode-se verificar que a densidade decai com 0 aumento das heterogeneidades por conta do tamanho médio do diâmetro das mesmas no modelo. A comparação das velocidades $\mathrm{P} / \mathrm{S}$ é bem satisfatório, pois na tabela as velocidades decaem em função do aumento das heterogeneidades e a análise nos casos secos e saturados que irão influenciar nas velocidades. Pode-se obter o gráfico das velocidades $P$ e $S$ para os modelos experimentais e teóricos nas Figuras 7, 8,9 e 10 .

A partir destes gráficos, podemos fazer a comparação de
Tabela 3: The measurements performed in-group $C$ of the heterogeneous models, performed in LPRP.

\begin{tabular}{ccccc}
\hline Group $B$ & $C-10$ & $C-20$ & $C-30$ & $C-40$ \\
\hline Lenght $(\mathrm{mm})$ & 54.36 & 57.13 & 56.91 & 56.98 \\
Mass $_{\text {dry }}(\mathrm{g})$ & 102.18 & 105.70 & 99.57 & 95.16 \\
Mass $_{\text {satured }}(\mathrm{g})$ & 116.06 & 120.57 & 114.59 & 111.07 \\
Porosity $\phi)$ & 0.232 & 0.237 & 0.240 & 0.254 \\
Volume $\left._{\left(\mathrm{cm}^{3}\right)}\right)$ & 59.62 & 62.66 & 62.42 & 62.49 \\
Density $_{\text {dry }}\left(\frac{\mathrm{g}}{\mathrm{cm}^{3}}\right)$ & 1.713 & 1.686 & 1.595 & 1.522 \\
Density $_{\text {sat }}\left(\frac{\mathrm{g}}{\mathrm{cm}^{3}}\right)$ & 1.946 & 1.924 & 1.835 & 1.777 \\
time $_{p \text { dry }}(\mathrm{s})$ & 20.27 & 21.95 & 22.41 & 23.06 \\
time $_{\text {s dry }}(\mathrm{s})$ & 29.68 & 32.63 & 35.01 & 37.57 \\
time $_{p \text { sat }}(\mathrm{s})$ & 17.79 & 19.32 & 20.14 & 21.04 \\
time $_{\text {s sat }}(\mathrm{s})$ & 31.46 & 34.99 & 37.36 & 40.20 \\
$\operatorname{Vp}_{\text {dry }}(\mathrm{m} / \mathrm{s})$ & 2663.23 & 2586.38 & 2523.70 & 2455.00 \\
$\operatorname{Vs}_{\text {dry }}(\mathrm{m} / \mathrm{s})$ & 1822.82 & 1743.46 & 1619.06 & 1511.00 \\
$\operatorname{Vp}_{\text {sat }}(\mathrm{m} / \mathrm{s})$ & 3031.60 & 2935.93 & 2806.21 & 2690.27 \\
$\operatorname{Vs}_{\text {sat }}(\mathrm{m} / \mathrm{s})$ & 1720.06 & 1626.27 & 1517.30 & 1412.41 \\
\hline
\end{tabular}

que a velocidade diminui de acordo com o aumento da porosidade, isso aconteceu na velocidade da onda $\mathrm{P}$ e S, com isso, comprovando os modelo empíricos de Han et al. (1986) e J. et al. (1986) que relacionam as velocidades de ondas ultrassônicas em relação a porosidade. Como pode ser observado nas figuras, as velocidade da onda $P$ decai mais rápido do que a velocidade de onda $S$ em relação ao aumento da porosidade Esse decaimento é mais acentuado para o grupo de amostra com maior número de espalhadores, pois há uma maior porcentagem de espaços vazios dentro das amostras. Esse decaimento tem uma maior variação quando a amostra está saturada, pois a água não suporta o cisalhamento, portanto, a velocidade da onda S é menor que a da onda P.

\section{Discussão e Conclusões}

Este trabalho mostra a importância da modelagem física para simulações de modelos isotrópicos heterogêneos. Como mencionado, estas amostras foram feitas baseadas na metodologia desenvolvida por Santos et al. (2017) para a fabricação de amostras porosas sintéticas (no contexto isotrópico e anisotrópico). Como foi observado, para as amostras do grupo $A, B$ e $C$, as velocidades da onda $\mathrm{P}$ e $\mathrm{S}$ foram bastante afetadas devido ao aumento da concentração de heterogeneidades dentro das amostras. A utilização dos dois modelos em relação aos dados experimentais foram bastante satisfatórios, tanto para o caso seco, quanto para o caso saturado, os modelos tiveram uma boa previsão para os grupos de amostras, e tiveram um erro percentual bastante baixo. Portanto, passando confiança para os modelos utilizados para a previsão das velocidades.

\section{Agradecimentos}

Gostaríamos de agradecer ao CNPq (459063/2014-6) e a PROPESP-UFPA pelo suporte financeiro. Também gostaríamos de agradecer ao PET-MEC-UFPA por ter proporcionado ajuda de custo na construção dos modelos.

\section{Referências}

Barwis, J. H., J. G. McPherson, and J. Studlick, 1990, Sandstone petroleum reservoirs: Spring Verlag.

Han, D., N. A., and M. D., 1986, Effects of porosity and clay 
content on wave velocities in sandstones.: Geophysics, 51, 2093-2107.

J., C., Batzle, M. Eastwood, and R. et al., 1986, Relationships between compressional wave and shearwave velocities in clastic silicate rocks.: Geophysics, 50, $571-581$.

S., R. J., and J. Korringa, 1975, On the dependence of the elastic properties of a porous rock on the compressibility of the pore fluid. geophysics: 40, 608-616.

Santos, L. K., D. F. J. J. S., and M. D. L. D. S. C. B, 2017, A new way to construct synthetic porous fractured médium: Journal of Petroleum Science and Eng., Manuscript under Revison.

Santos, L. K., D. F. J. J. S., and C. B. Da Silva, 2016, A study of ultrasonic physical modeling of isotropic media based on dvnamic similitude: Ultrasonics. 70. 227-237.

Effective P Velocity(DRY and SAT-Group A)-Maxwell-Garnett(MG) and Kuster-Toksoz(KT)

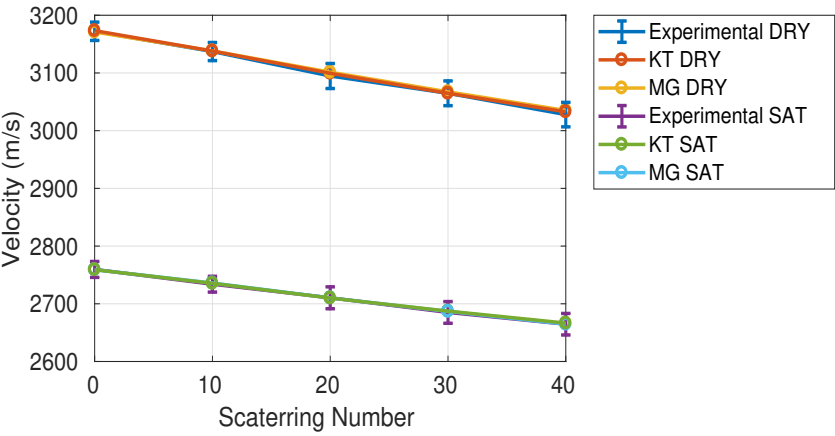

Figura 5: The velocity of the $P$ wave of group $A$ in relation to the number of heterogeneities for the dry and saturated cases with error percentage for each sample data.

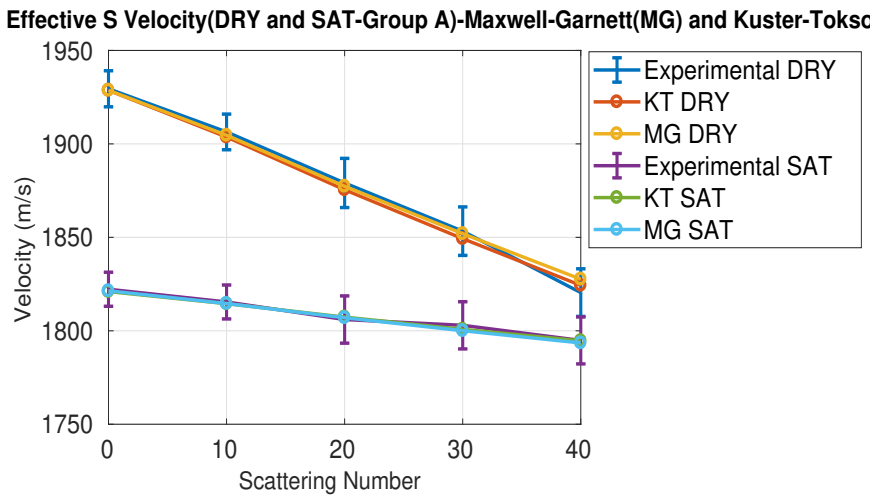

Figura 6: The velocity of the $S$ wave of group $A$ in relation to the number of heterogeneities for the dry and saturated cases with error percentage for each sample data.

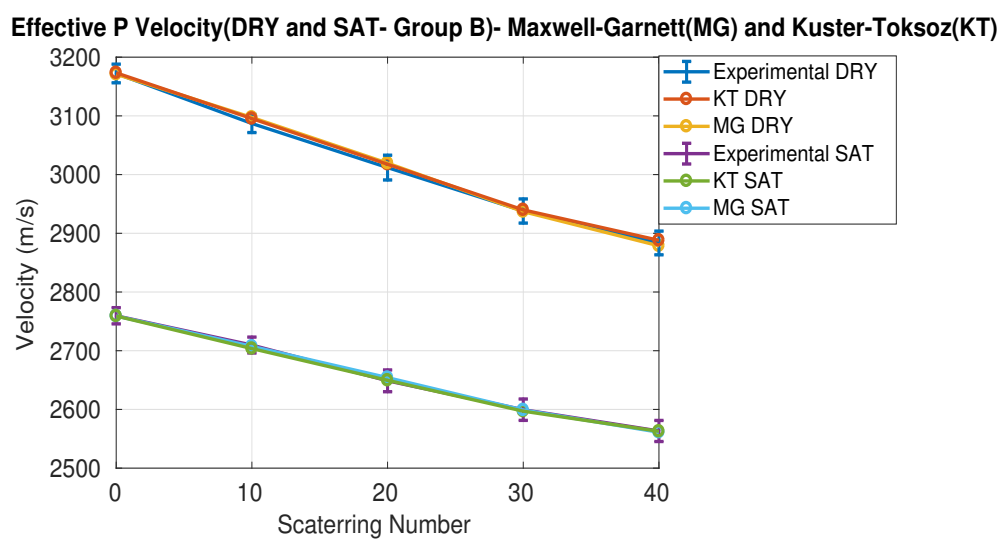

Figura 7: The velocity of the $\mathrm{P}$ wave of group $\mathrm{B}$ in relation to the number of heterogeneities for the dry and saturated cases with error percentage for each sample data.

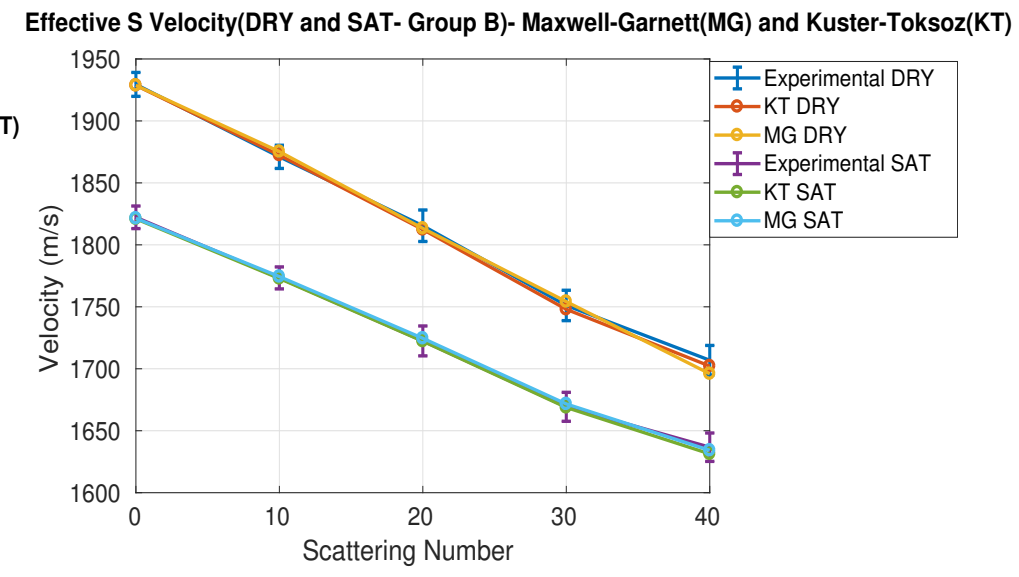

Figura 8: The velocity of the $S$ wave of group $B$ in relation to the number of heterogeneities for the dry and saturated cases with error percentage for each sample data. 
Effective P Velocity(DRY and SAT- Group C)- Maxwell-Garnett(MG) and Kuster-Toksoz(KT)

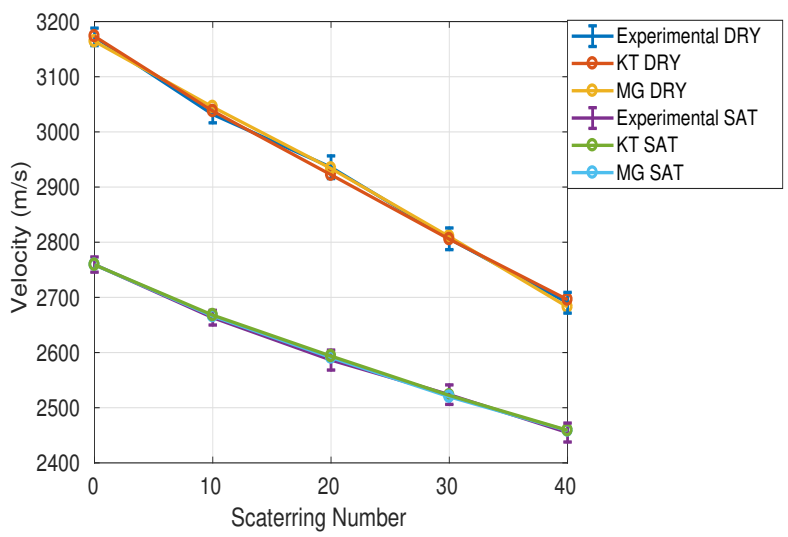

Figura 9: The velocity of the $P$ wave of group $C$ in relation to the number of heterogeneities for the dry and saturated cases with error percentage for each sample data.

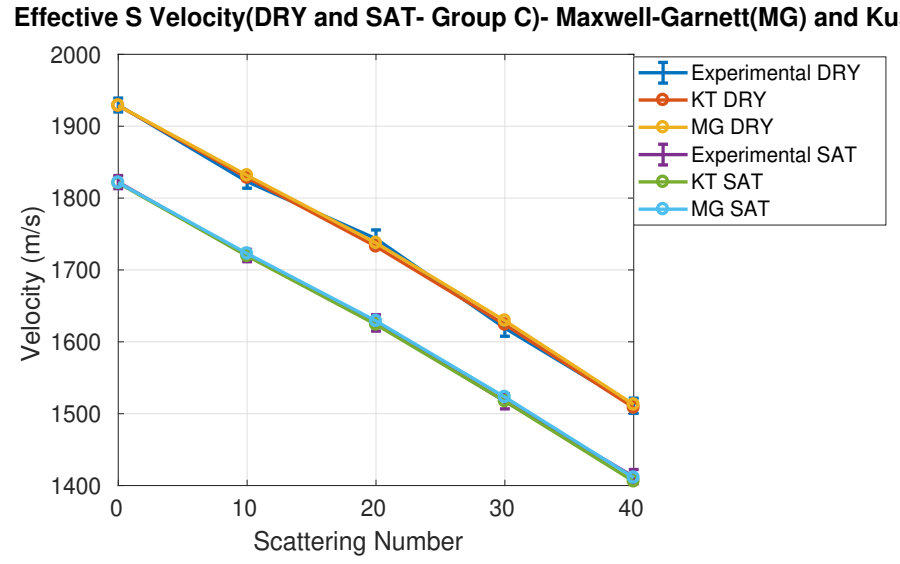

Figura 10: The velocity of the $S$ wave of group $C$ in relation to the number of heterogeneities for the dry and saturated cases with error percentage for each sample data.
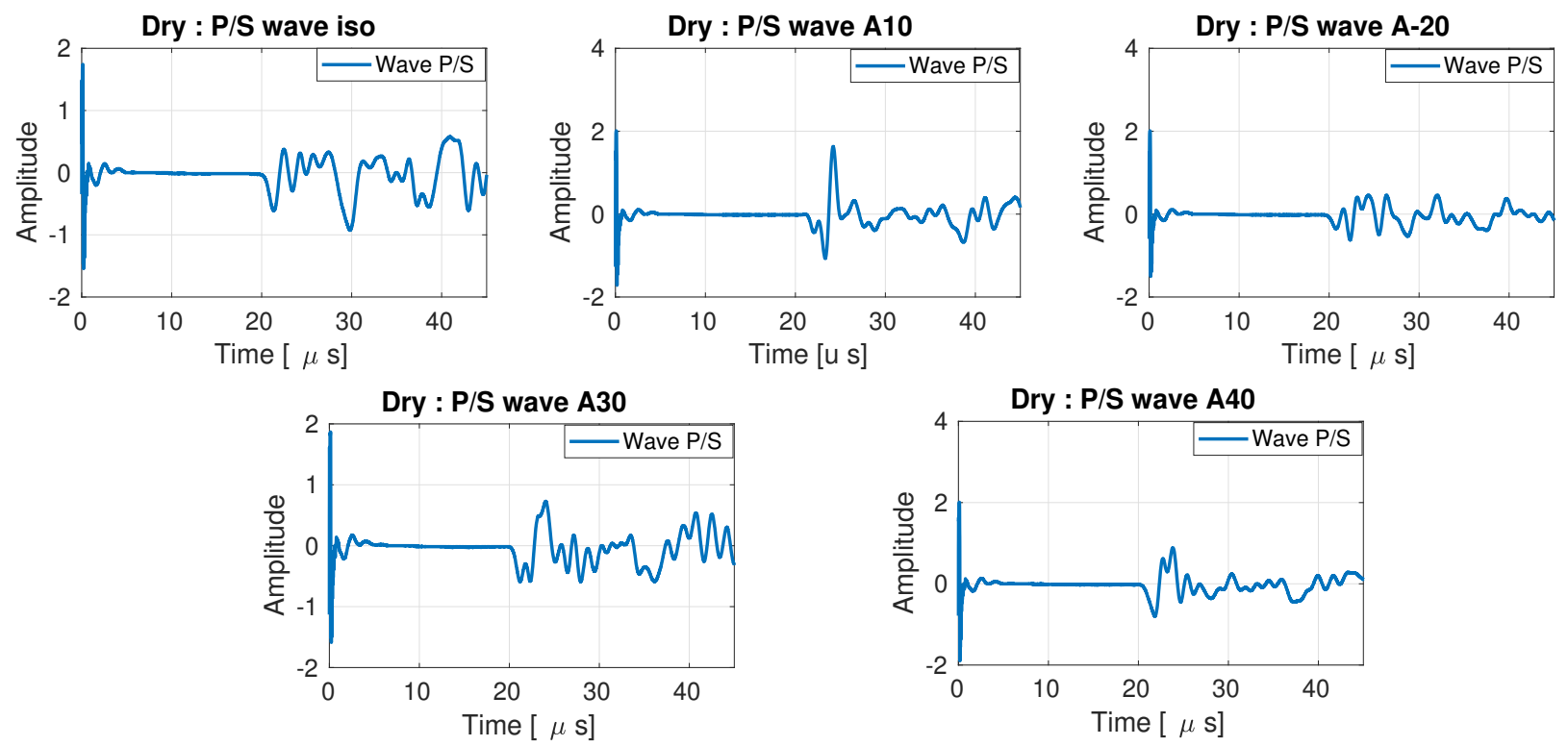

Figura 11: Seismic traces obtained from samples $A$ and isotropic with this the velocities $P$ and $S$ will be obtained. 